\title{
Anatomie
}

\section{des glandes salivaires principales. Application à la chirurgie de la glande sublinguale}

\section{RÉSUMÉ}

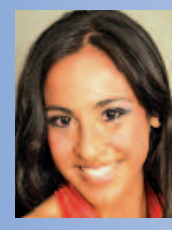

Rivka BENDRIHEM

Faculté de Médecine Paris-Descartes (Paris V).

\section{Christian VACHER}

Service de Chirurgie maxillo-faciale et Stomatologie,

Hôpital Beaujon,

AP-HP et Faculté de Médecine Paris-Diderot (Paris VII)

100, boulevard du Général Leclerc 92118 Clichy cedex.

Les glandes salivaires principales sont classiquement situées dans le plancher de la bouche pour la glande sublinguale, dans la région submandibulaire pour la glande submandibulaire et dans la région parotidienne pour la parotide. En réalité, il n'existe pas de barrière anatomique nette entre ces différentes glandes salivaires qui semblent réaliser une solution de continuité entre ces trois régions anatomiques. Ceci explique que le diagnostic de localisation des tumeurs salivaires est parfois difficile. L'abord chirurgical des zones frontières est également compliqué en cas de tumeur salivaire du plancher buccal latéral, qui peut être due soit à une tumeur du prolongement oral de la glande submandibulaire, soit à une tumeur de la glande sublinguale. Nous donnons en exemple l'observation d'une patiente qui présentait une volumineuse tumeur de la glande sublinguale (carcinome adénoïde kystique), dont l'exérèse n'a pu être réalisée que par un abord combiné oral et cervical.

- glande sublinguale

- glande submandibulaire

- glande parotide

- carcinome adénoïde kystique

- chirurgie 


\section{Introduction}

$>$ Les glandes salivaires principales sont classiquement décrites somme des glandes situées dans des loges anatomiques individualisées [1, 2]. On pourrait donc imaginer que le diagnostic de localisation d'une tumeur d'une glande salivaire principale est simple à effectuer quand on sait que tout nodule situé dans la région parotidienne, dans la région submandibulaire ou sublinguale doit faire évoquer une tumeur salivaire jusqu'à preuve du contraire [3]. En réalité, il est parfois difficile de faire le diagnostic entre une tumeur du pôle inférieur de la parotide et une tumeur de la partie postérieure de la glande submandibulaire. C'est pourtant essentiel car la voie d'abord d'une parotidectomie est une voie de "lifting " pré-auriculaire poursuivie

\section{Matériel et méthode}

\section{Cas clinique}

Une patiente âgée de 79 ans s'est présentée à la consultation de chirurgie maxillo-faciale pour une augmentation de volume de la région submandibulaire droite et du plancher buccal antéro-latéral gauche. L'examen clinique a mis en évidence un nodule dur de $3,5 \mathrm{~cm}$ de grand axe, bien limité, à la palpation de la région submandibulaire saillant dans le plancher buccal antéro-latéral droit. Ce nodule n'adhérait pas à la peau et aux plans profonds, il était mobile et indolore. Un scanner cervicofacial injecté a été effectué qui confirme le diagnostic de tumeur du plancher buccal développé soit à partir de la glande submandibulaire dans son prolongement oral, soit de la glande derrière l'oreille au niveau de la racine des cheveux, alors que la voie d'abord de la glande submandibulaire est une voie cervicale horizontale. De la même manière, il est parfois difficile devant une tumeur du plancher buccal latéral d'en attribuer l'origine soit à la glande sublinguale soit à la glande submandibulaire. Là encore, la voie d'abord peut être différente, plutôt orale pour la glande sublinguale, alors qu'elle doit être cervicale pour la glande submandibulaire. Le but de cet article est de présenter une observation de tumeur salivaire sublinguale et de préciser l'anatomie de ces deux zones frontières en illustrant les communications qui existent entre les trois espaces occupés par des glandes salivaires principales.

sublinguale (fig. 1). Une exérèse chirurgicale sous anesthésie générale a alors été décidée, par voie orale de première intention. Lors de I'installation de la patiente, la région cervicale a été laissée dans le champ opératoire de manière à permettre un abord cervical en cas de besoin. Au cours de l'intervention, après un abord par voie orale du plancher buccal latéral (fig. 2), il est apparu que l'abord strictement oral empêchait de contrôler la totalité de l'exérèse de la lésion qui se prolongeait dans la région sub-mandibulaire. Une incision cutanée horizontale située deux travers de doigt sous le bord basilaire de la mandibule a été effectuée, ce qui a permis de libérer la tumeur en totalité et de l'extraire par voie orale (fig. 3). La voie d'abord du plancher a 
été suturée par du fil résorbable, la voie cervicale a été fermée en deux plans sur un drain aspiratif. Les suites opératoires ont été simples. L'analyse anatomo-pathologique de la pièce a conclu à un carcinome adénoïde kystique dont l'exérèse était complète. Le dossier
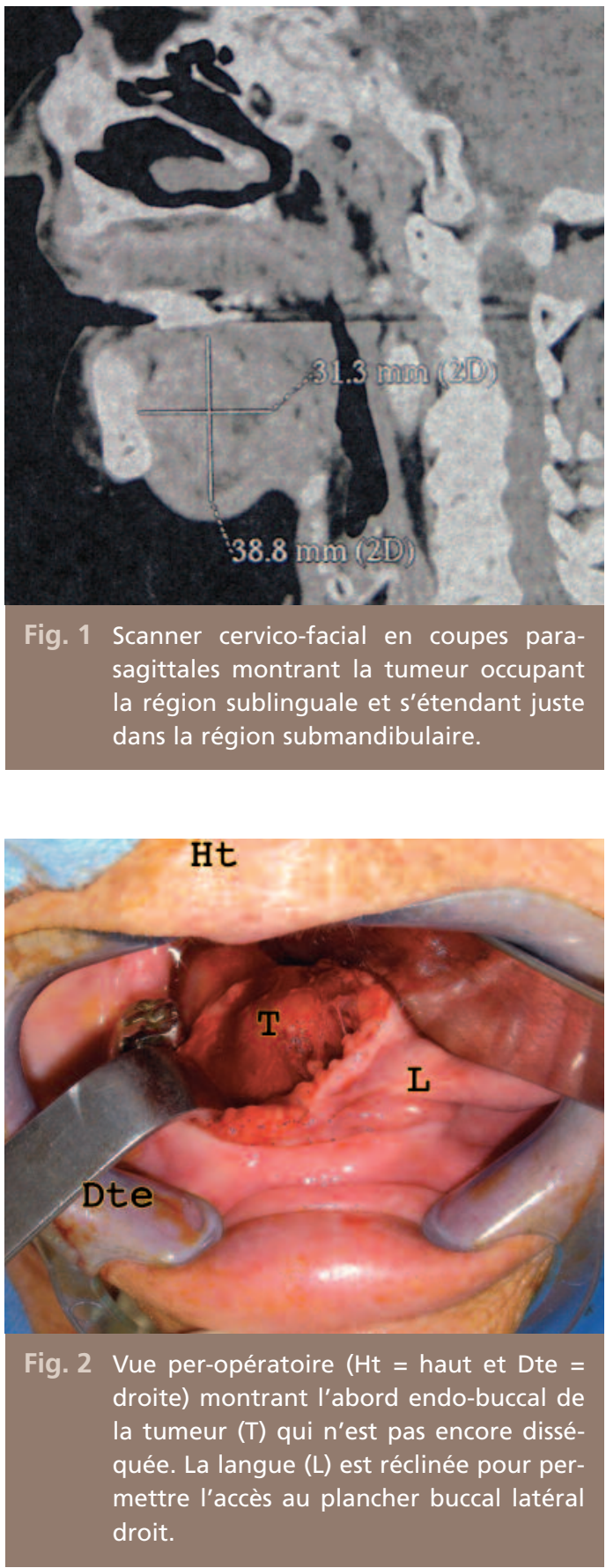

de cette patiente a été présenté en Réunion de concertation pluridisciplinaire (RCP). Après avis des oncologues et radiothérapeutes, en raison de la taille importante de la tumeur et malgré le caractère complet de l'exérèse, il a été décidé de proposer une radiothérapie complémentaire à cette patiente. Celle-ci l'a refusée malgré l'insistance de l'ensemble de l'équipe. Une surveillance a été décidée avec une consultation tous les trois mois la première année et tous les six mois ensuite sans limitation de durée. Un scanner cervico-facial et thoracique a été programmé tous les ans pour dépister une éventuelle récidive locale inaccessible à l'examen clinique et d'éventuelles métastases pulmonaires qui constituent le risque le plus important de dissémination à distance. À deux ans postopératoires, la patiente n'a présenté aucune récidive, sa surveillance se poursuit.

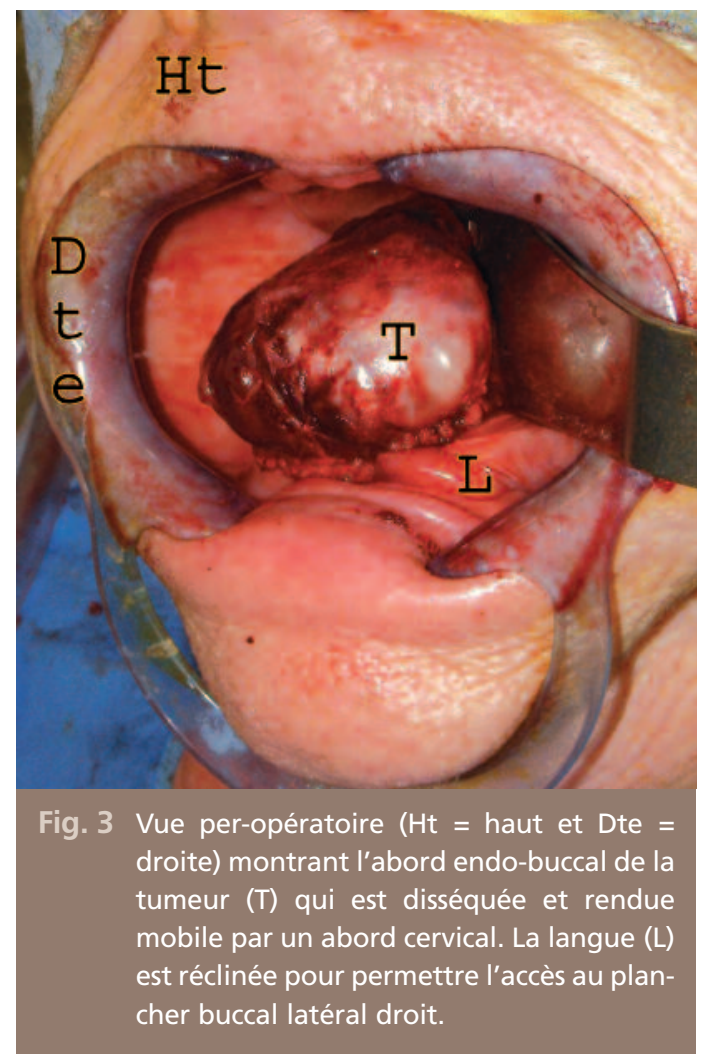


Pour mettre en évidence les communications qui existent entre région submandibulaire et sublinguale et entre région parotidienne et submandibulaire, il a été effectué une dissection anatomique de la région submandibulaire

\section{Résultats}

Chez tous les sujets qui ont fait l'objet d'une dissection anatomique, il a été observé qu'il n'existait aucune structure séparant la glande submandibulaire de la parotide en arrière et de la glande sublinguale en avant. L'étude d'anatomie radiologique des IRM a permis d'aboutir à la même conclusion dans tous les cas. La glande submandibulaire était au contact avec la glande sublinguale au niveau du plancher buccal antéro-latéral dans tous les cas au niveau de son prolongement oral qui se chez cinq sujets anatomiques frais, et cinq IRM de patients ne présentant pas de pathologie salivaire ont été étudiées afin de déterminer $s^{\prime} i l$ existe des limites anatomiques nettes entre les trois glandes salivaires. place médialement par rapport au pôle postérieur de la glande sublinguale. La glande parotide, quand elle était volumineuse, venait au contact du pôle postérieur de la glande submandibulaire (fig. 4). Quand la parotide était de petite taille elle n'était pas directement en contact avec la glande submandibulaire (fig. 5) mais elle n'en était séparée par aucun élément anatomique dans sa partie la plus superficielle sous cutanée en dessous de l'angle mandibulaire.
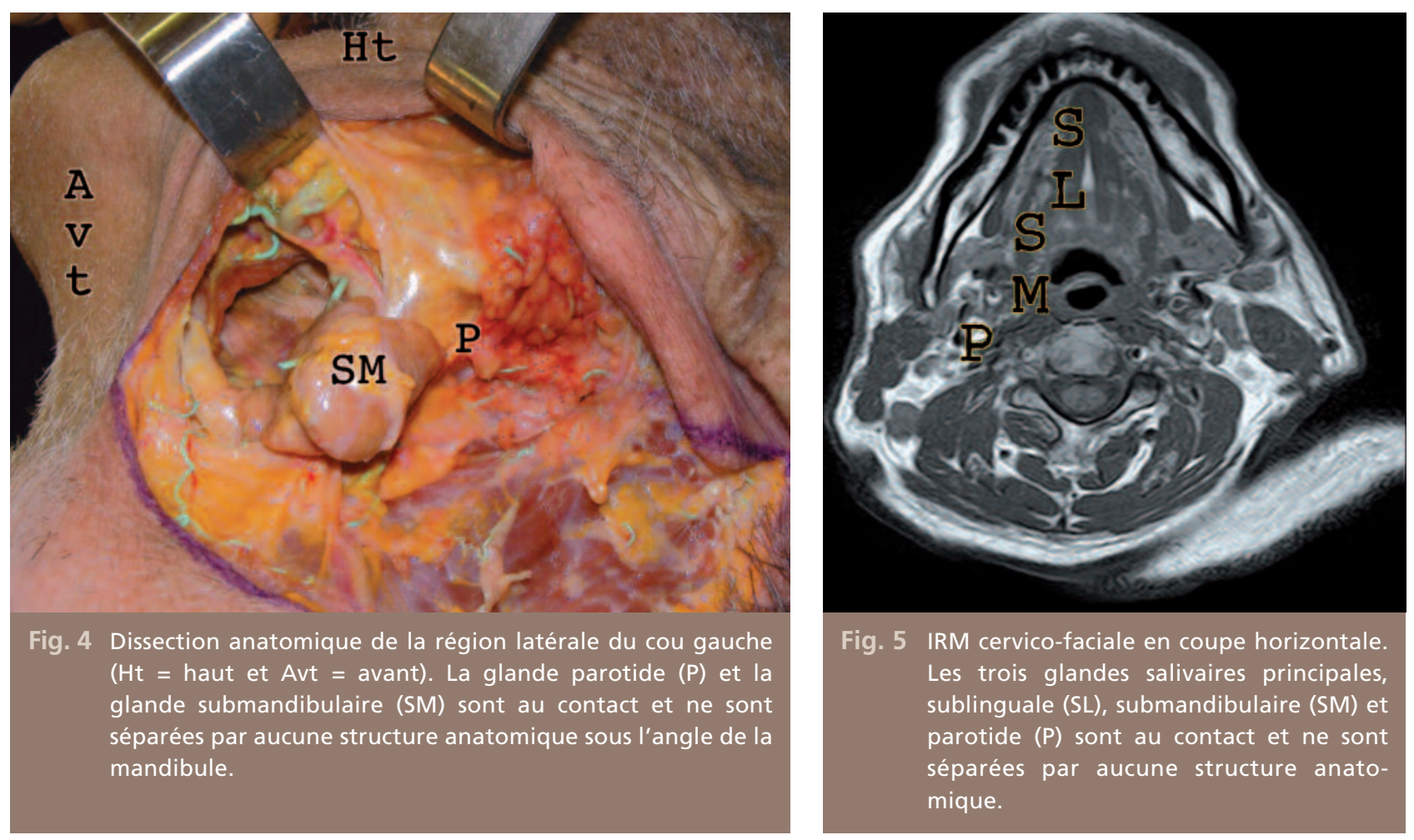


\section{Discussion}

Le carcinome adénoïde kystique est une tumeur maligne salivaire rare qui a tendance à infiltrer les nerfs [3]. Cependant c'est la forme histologique la plus fréquente des tumeurs de la glande sublinguale $[4,5,6]$. Le traitement de première intention de ces tumeurs est l'exérèse chirurgicale large [6]. Il peut être complété par une radiothérapie postopératoire lorsque la tumeur est très volumineuse, que les marges d'exérèse sont insuffisantes $[4,6]$. La chimiothérapie n'est que peu efficace sur ces tumeurs jusqu'à ce jour [6]. Ces tumeurs sont peu lymphophiles et il n'y a pas de consensus sur la nécessité de la réalisation d'un curage cervical [3]. L'évolution des carcinomes adénoïdes kystiques est marquée par le risque de récidive locale et surtout de métastases pulmonaires qui, dans certains cas peuvent rester asymptomatiques pendant de longues années [6]. Le caractère complet de l'exérèse initiale est donc particulièrement important même en cas de métastases pulmonaires. La surveillance clinique et radiologique du risque de récidives et de l'apparition de métastases pulmonaires est essentielle.

Cette observation et notre étude montrent qu'il existe un véritable espace salivaire allant depuis la parotide située en dehors du ramus mandibulaire jusqu'au plancher buccal antéro-latéral et passant par la région submandibulaire (fig. 6). Comme nous l'avons montré, la loge parotidienne communique avec la loge submandibulaire au niveau de la partie la plus inférieure (sous l'angle mandibulaire) et la plus superficielle de la région parotidienne. Au-dessus de l'angle mandibulaire, les deux loges sont séparées par le muscle styloglosse et le ligament stylomandibulaire [7]. II est classique de décrire une séparation des deux loges en dessous de l'angle mandibulaire par la bandelette mandibulaire qui est un tractus fibreux tendu entre le fascia du muscle sterno-cleido-mastöidien et l'angle mandibulaire. Au cours des dissections, nous

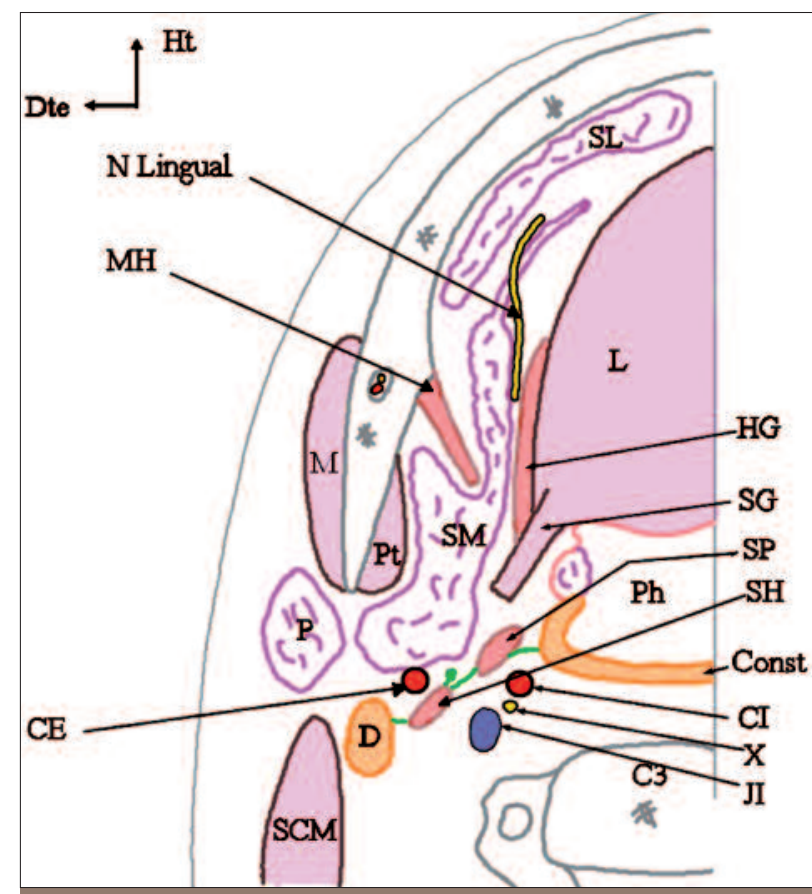

Fig. 6 Coupe anatomique axiale passant par C3. Les trois glandes salivaires principales, la glande parotide $(\mathrm{P})$, la glande submandibulaire (SM) et la glande sublinguale (SL) sont situées dans un défilé anatomique. Entre les glandes submandibulaire et sublinguale il existe un défilé anatomique limité latéralement par les muscles mylo-hyoïdien (MH) et hyo-glosse (HG), muscle le plus latéral de la langue ( $\mathrm{L}$ ) dans lequel passe également le nerf lingual ( $\mathrm{N}$ Lingual). Les muscles présents sur cette coupe sont : SCM = sterno-cleïdo-mastoïdien, $\mathrm{D}=$ digastrique, $\mathrm{SH}=$ stylo-hyoïdien, $\mathrm{SG}=$ styloglosse, SP = stylo-pharyngien, $\mathrm{M}=$ masseter, $\mathrm{Pt}=$ ptérygoïdien interne, Const = constricteur supérieur du pharynx (Ph). Les vaisseaux sont : $\mathrm{Cl}=$ artère carotide interne, $C E=$ artère carotide externe, $\mathrm{JI}=$ veine jugulaire interne. $X=$ nerf vague. 
n'avons jamais observé de bandelette mandibulaire. Cette bandelette ne figure pas dans la nomenclature anatomique internationale, elle n'est présente que dans les descriptions anatomiques en français et il paraît légitime de s'interroger sur sa réalité. Ainsi une tumeur du pôle inférieur de la glande parotide peut parfaitement être confondue avec une tumeur de la glande submandibulaire, surtout si la patient présente de volumineuses parotides. Pourtant l'abord chirurgical est différent, puisqu'une parotidectomie doit être faite avec une dissection du nerf facial depuis son origine au niveau du foramen stylo-mastoïdien alors qu'une submandibulectomie se fait par voie cervicale. De même, comme le montre l'observation présen- tée, une tumeur de la glande sublinguale doit être abordée logiquement par voie orale, alors qu'une submandibulectomie ne peut être effectuée par cette voie d'abord. Cependant, une voie d'abord combinée peut être utile, par exemple en cas de tumeur sublinguale très volumineuse comme nous l'avons montré, et dans certains cas de tumeur sublinguale envahissant le conduit submandibulaire [8]. De la même manière, lorsque qu'une submandibulectomie est indiquée pour de multiples lithiases canalaires et glandulaires, il peut être utile d'aborder le canal de la glande dans le plancher buccal ou de la cathétériser pour éviter de laisser une lithiase canalaire en fin d'intervention.

\section{Bibliographie}

1. Drake RL, Vogl W, Mitchell AWM.

Gray's anatomy for students.

Edition française :

Duparc J, Duparc F.

Anatomie pour

les étudiants.

Paris : Elsevier, 2006.

2. Norton $\mathrm{SN}$, Netter FH.

Édition française : Duparc F.

Précis d'anatomie clinique

de la tête et du cou.

Paris : Masson, 2009.

3. Vaillant JM, Laudenbach P.

Pathologie

des glandes salivaires.

Paris : Flammarion, 1988.

4. Zdanowski $\mathrm{R}$, Dias $\mathrm{FL}$, Barbosa MM, Lima RA,
Faria PA, Loyola AM,

Nascimento Souza KC.

Sublingual gland tumors:

clinical, pathologic,

and therapeutic analysis

of 13 patients treated

in a single institution.

Head Neck

2011 Apr;33(4):476-81.

5. Sun $G$, Yang $X$, Tang $E$, Wen J, Lu M, Hu Q.

The treatment of sublingual gland tumors. Int J Oral Maxillofac Surg 2010 Sep;39(9):863-8.

6. Yu T, Gao Qh, Wang XY, Wen YM, Li LJ.

Malignant sublingual gland tumors: a retrospective clinicopathologic study

of 28 cases.

Oncology 2007;72(1-2):39-44.

Epub 2007 Nov 12.

7. Gaudy JF, Vacher C.

Atlas d'anatomie clinique

et chirurgicale des tissus

superficiels de la tête

et du cou.

Paris : Elsevier Masson, 2010.

8. Saito $M$, Nishiyama $H$, Maruyama S, Oda Y, Saku T, Hayashi T.

Adenoid cystic carcinoma

of sublingual gland

involving

the submandibular duct.

Dentomaxillofac Radiol

2008 Oct;37(7):421-4. 


\section{SUMMARY}

\section{Anatomy of major salivary glands}

Rivka BENDRIHEM, Christian VACHER

$$
\begin{aligned}
& \text { Keywords } \\
& \text { - sublingual gland } \\
& \text { - submandibulary gland } \\
& \text { - parotid gland } \\
& \text { - adenoid cystic } \\
& \text { carcinoma } \\
& \text { - surgery }
\end{aligned}
$$

\begin{abstract}
The major salivary glands are usually considered as located in different spaces: the sublingual gland in the oral floor, the submandibular gland in the submandibular area and the parotid gland in the parotid area. But in the truth, there is no anatomic separation between these different salivary glands which are located in continuity along these anatomic areas. This is the reason why the diagnosis of localization of salivary tumors can be sometimes difficult. The surgical access of these frontier areas can be also difficult in case of salivary tumor of the lateral oral floor, which origin can be the oral part of the submandibular gland or a sublingual gland tumor. We describe the observation of a patient who presented a sublingual gland tumor (adenoid cystic carcinoma) which has been removed by a combined oral and cervical access.
\end{abstract}

\section{Apprenez à respirer à vos enfants}

Jean-Paul Allaux

Les maladies ORL avec leur prolongement au niveau pulmonaire sont malheureusement de plus en plus répandues de nos jours et à tout âge.

La pollution atmosphérique, les appartements surchauffés ou enfumés par des parents tabagiques, les rues des villes saturées de gaz d'échappement... tous ces maux contemporains bien connus sont bien souvent responsables de l'installation de ces affections dès la petite enfance. Ils freinent par ailleurs le développement harmonieux du corps en installant parfois des déformations vertébrales mais aussi du palais et des mâchoires provoquant une mauvaise implantation des dents.

Fruit d'une longue expérience, ce livre se veut avant tout pratique. De nombreux exercices spécifiques à faire à la maison ou à l'école, seul ou en groupe, permettront aux éducateurs, parents et enseignants de trouver remède à ces différentes affections et d'aider efficacement les enfants à s'épanouir pleinement.

Un livre pour apprendre à respirer à nos enfants.

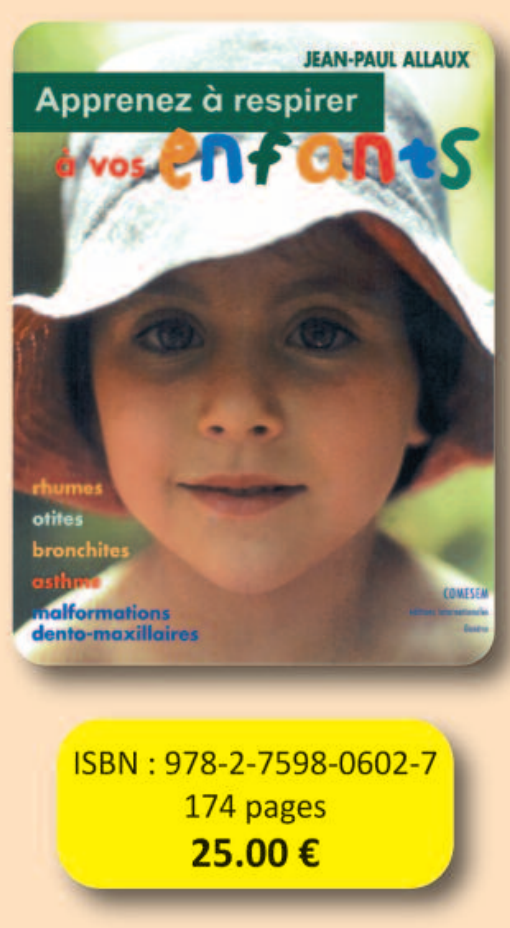

174 pages

$25.00 €$

\section{Commandez directement sur : www.edition-sciences.com}

